ORIGINAL ARTICLE

\title{
Comparison of BRACE (balance, resistance, aerobic, cognitive exercises) and proprioceptive training on balance and mobility in knee osteoarthritis.
}

\author{
Ali Raza', Khadija Liaquat ${ }^{2}$, Hafiz Salman Saeed ${ }^{3}$
}

Article Citation: Raza A, Liaquat K, Saeed HS. Comparison of BRACE (balance, resistance, aerobic, cognitive exercises) and proprioceptive training on balance and mobility in knee osteoarthritis. Professional Med J 2022; 29(3):303-309.

https://doi.org/10.29309/TPMJ/2022.29.03.6833

ABSTRACT... Objective: The purpose of the study was to find the effect of Balance, Resistance, Aerobic, Cognitive Exercise (BRACE) and proprioceptive training on balance and mobility in knee osteoarthritis. Study Design: Randomized Controlled Trial. Setting: Independent University Hospital Faisalabad. Period: September 2020 to December 2020. Material \& Methods: A sample of 18 participants were included. The participants were divided into 2 groups BRACE and proprioceptive training by lottery method. The participants got 3 training sessions per week for 8 weeks. The measurements were taken at baseline and after 8 weeks. Statistical Package for Social Sciences(SPSS 27) was used to analyzed the data. Results: The age of participants in both groups was $(55.67 \pm 2.761)$ years. The gender distribution of participants was female $(55 \%)$ and male (45\%). Within group analysis shows statistically significant relat ion on BBS and TUG from baseline to 8 weeks in both groups $(p<0.05)$, On CTSIB frim eyes closed and foam eyes closed show significant difference in both groups ( $<<0.05)$. Between group analysis shows that no statistically significant relation in both groups ( $p>0.05)$. Conclusion: Both groups shows comparable improvements in balance and mobility but BRACE protocol showed more improvement as compared with proprioceptive.

Key words: Berg Balance Scale, BRACE Protocol, Knee Osteoarthritis, Proprioceptive Training.

\section{INTRODUCTION}

Osteoarthritis (OA) of knee is most public health problem referred by discomfort and decrease in functional activities. The incidence of symptomatic knee $\mathrm{OA}$ is $13 \%$ in female and $10 \%$ in male. The second most common cause of disability among older adults is OA. ${ }^{1}$ Characteristic of OA is degenerative and progresses with age that can lead to muscle weakness, pain and functional limitations for example joint stiffness reduced activity duration of less than 30 minutes. ${ }^{2}$ Physical therapy can contribute to decrease in pain, muscle strength, improved proprioception, postural stability, increased independence in activities of daily living and reduction of depression symptoms. ${ }^{3}$ Balance is a important component of functions which allows persons to maintain their position and respond to perturbations. Dynamic balance comprises of balance control with movements and significant aspects of whole balance skills. A risk factors for fall in participants with knee osteoarthritis were reduced balance control. ${ }^{4}$

Proprioception can be defined as a conscious, unconscious and semiconscious awareness or sense about position, force, velocity and movement of an extremity, limb or joint in space. The Proprioception of knee joint declined with increasing age and with the presence of progression knee OA. ${ }^{5}$ Proprioception responsible to provide primary information for balance and extent through skin, muscles, ligaments and tendons. Postural sway can also affect due to damage in lower limb proprioception. Proprioceptive recovery is an essential factor through out therapy for patients. ${ }^{6}$ Regulation of balance depends on the proprioceptive, visual
1. DPT MSPT (NM), Physiotherapist, IUH Faislabad.

2. DPT, MSPT (NM), Senior Lecturer, RCR AHS, Ripha University Lahore.

3. MBBS, FCPS, FCPS, Associate Professor Orthopedic, IUH Faisalabd.

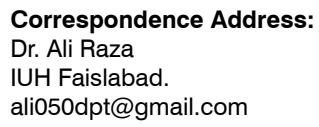

Correspondence Address:

Dr. Ali Raza

IUH Faislabad.

ali050dpt@gmail.com

Article received on:

Accepted for publication:
$30 / 09 / 2021$ $05 / 01 / 2022$ 
and vestibular stimuli. The reduction in balance control may lead to fall injuries that result in serious psychological, social and physical loss. In knee OA patients an effective assessment of balance control is necessary before management of condition. ${ }^{7}$

Aslihan in 2020 compared effect of performance of single versus dual task exercise on balance in elderly persons with knee osteoarthritis (KOA). They concluded that dual task exercises was not superior to single task exercises for improving balance in knee OA patients. ${ }^{7}$ In 2019 Gulsen reported that greater numbers of falls in older people with knee osteoarthritis (KOA) has been experienced by participants with no OA. Balance tests were applied with Tetrax Interactive Balance System, Berg Balance Scale (BBS) was applied formeasured the balance of patients. He concluded that appropriate physiotherapy and rehabilitation programme for knee osteoarthritis patients helps to improv balance and reduces risks of falling ${ }^{8}$ Ferraz et $\mathrm{Al}$ in 2018 assessed the influence of a low intensity exercises related with incomplete blood flow limitation in individuals with KOA. Blood flow ressitance training (all $p<0.05$ ). Significantly blood flow resistance training were also able for improved pain while encouraging less joint stress developed a effective therapeutic in KOA management. ${ }^{9}$

In literature many techniques was available like electrotherapy, manual therapy and some instrumental based techniques was reported which measured effects on balance and mobility in knee OA. I want to introduce performance based exercises for physiotherapist to improve balance and mobility which was performed at clinical setup as well as home base program. There was a lot of researche articles published specifically on balance training exercises, resistance exercises and aerobic exercises separately. Previously supervised BRACE protocol was used on mobility to reduce risk of fall. Now I used BRACE and Proprioceptive training for improve balance and mobility in elderly with knee OA. The Objective of this study was to compare the effect of BRACE and proprioceptive training on balance in knee osteoarthritis and to compare the effect of BRACE and proprioceptive training on mobility in knee osteoarthritis.

\section{MATERIAL \& METHODS}

The study design was Randomized Controlled Trial. The study was conducted in Physical Therapy Department of Independent University Hospital Faisalabad from September 2020 to December 2020. The study was completed in 6 months. A sample of 18 is calculated by Epitool. ${ }^{10}$ A $20 \%$ attrition rate is added and sample is 22 , 11 in each. Group A: BRACE protocol training and Conventional physical therapy, Group B: Proprioceptive training and Conventional physical therapy The convenient sampling technique was used to collect the data.

\section{Inclusion Criteria}

1. Age 50-70 years.

2. Both Male and Female

3. Knee osteoarthritis unilateral and bilateral. ${ }^{11}$

4. American College of Rheumatology (ACR) criteria for identifying osteoarthritis of knee joint is occurrence of discomfort in knee joint and any three of six factors listed below. ${ }^{12}$

1. Age 50y or more

2. Occurrence of crepitus sound on active motion

3. Morning stiffness less than $\mathbf{3 0} \mathrm{min}$

4. Bony inflammation

5. Bone overgrowth

6. No palpable warmness of synovium

7. Mini-mental state examination (MMSE) 23 or higher. ${ }^{13}$

8. Berg Balance Scale (BBS) $30-45$ points. ${ }^{14}$

\section{Exclusion Criteria}

Patients were exclude because they exhibit;

1. History of stroke during the previous 12 months

2. History of cardiovascular or musculoskeletal diseases,

3. Previously used medication for the central nervous system.

4. Presence of lower limb lesion or fracture in the last six months. ${ }^{15}$ 


\section{Data Collection Tool}

1. Berg Balance Scale (BBS) have shown high intrarater and interrater reliability (ICC $0.98)$ and Intra Class Coefficient $(I C C=0.97$ $0.98)^{14,16}$

2. Time Up and Go test (TUG) show excellent reliability (ICC>.95). ${ }^{17}$

3. Modified Clinical Test of Sensory Interaction in Balance (CTSIB). ${ }^{18}$

\section{Interventional Protocol (Conventional Treatment)}

Participants was received following treatment, heat treatment by infrared lamp which was positioned about $30 \mathrm{~cm}$ from the knee surface for almost 15- 20 minutes, strengthening of selected muscles specially quads muscles (isometric and dynamic). ${ }^{19}$

\section{Brace Protocol ${ }^{20}$}

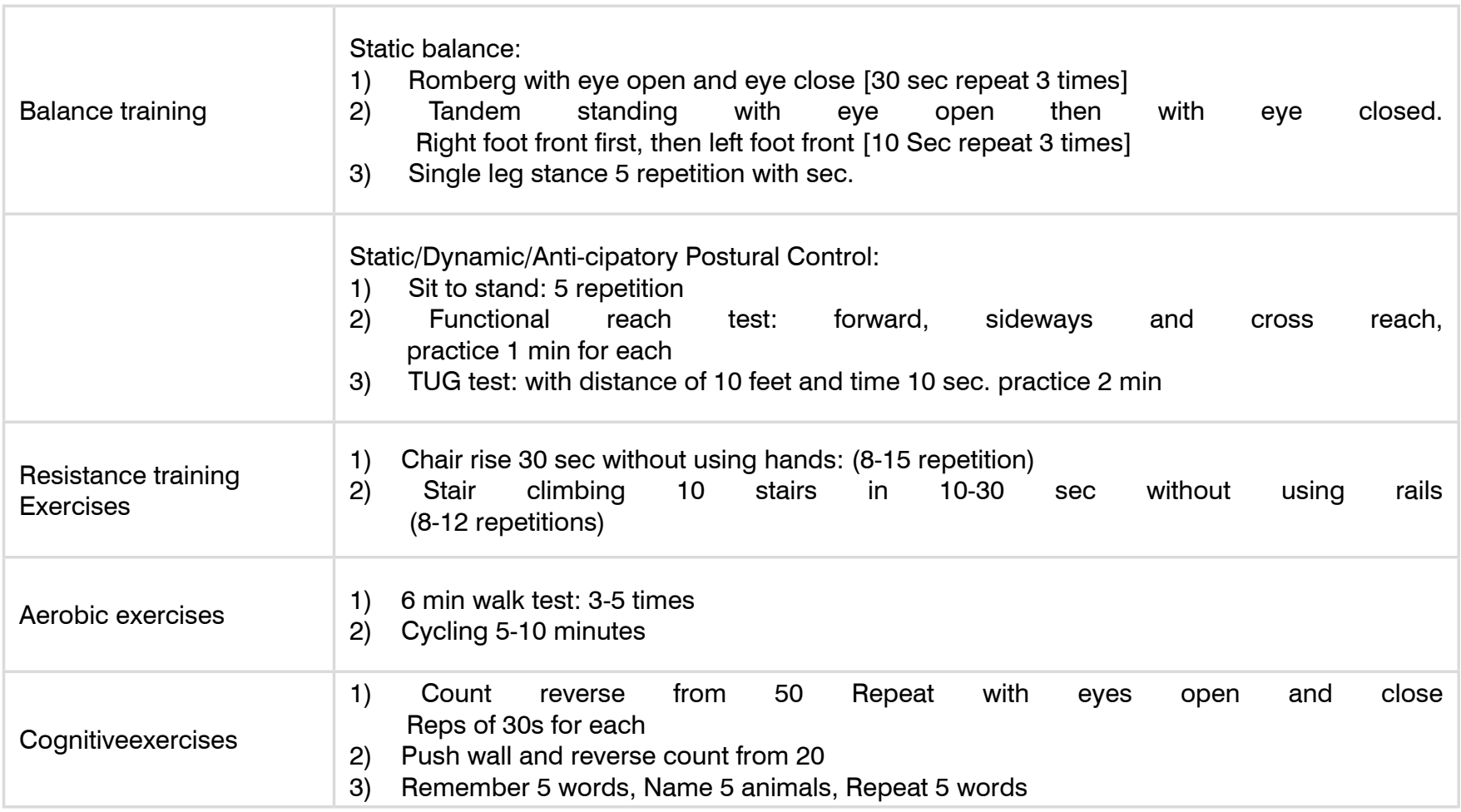

\section{Proprioceptive Training Phase ${ }^{6}$}

$1^{\text {st }}$ Phase - Steady phase

( $1^{\text {st }}$ set: Eyes Open, $2^{\text {nd }}$ set: Eyes Close)

Preserve stand up position

Equally heels up and down

Preserve stand up on one leg by turns

$2^{\text {nd }}$ Phase - Dynamic phase

Standing training on unbalanced

( $1^{\text {st }}$ set with open eyes and $2^{\text {nd }}$ set with closed eyes)

Preserve stand up position

Equally heels up and down

Preserve stand up on one leg by turns

Take step by turns

$3^{\text {rd }}$ Phase - Functional phase

Walking on the equal place

Equally feet together, stand up with back and forward foot position

Siting down $\rightarrow$ Squating position $\rightarrow$ Standing up
For 4 minutes per set

For 10s (5times per set)

For 10s (5times per set)

For 10s (5times per set)

For 6 minutes per set

For 10s (5times per set) For 10s (5times per set) For 10s (5times per set) For 10s (5times per set)

For 10 minutes

For 6 minutes

For 10s (5times per set)

For $1 \mathrm{~min}$ 
Informed Consent was signed. Used Standardized Questionnaire to collect the data. 8 weeks of interventional was provided 3 sessions per week for 8 weeks. The data was collected at baseline and after 8 weeks. Randomization was done by lottery method without replacement. Odd numberwas allocated to Group A and even number was allocated to Group B. Data was analyzed through SPSS 27. Data was checked for its normality. Data was normally distributed parametric test applied, within groups comparison paired sample T-test, between groups independent sample T-test was applied. Ethical clearance certificate had taken from institutional research board committee (REC/RCRS/20/2036.

\section{RESULTS}

On the basis of inclusion and exclusion criteria total 18 participants were recruited in this trial. Independent sample T-test was used to assess significance difference across the groups. Paired sample $\mathrm{T}$ test was used measure difference within the groups. For statistically significance $p$ value $(<0.05)$ or less and not significance $p$ value $(>0.05)$ or more selected. Age of the participants after Sequential Deletion total number of participants was $(\mathrm{N}=18)$, mean of age (56.28) and standard deviations (SD) (2.585). Gender distribution in Group A 55\% male and 45\% female were participated. Gender distribution in Group B $36 \%$ male and $64 \%$ female were participated.

Within group analysis paired sample $T$ test was applied and show statistically significant relation on BBS and TUG in both groups ( $p<0.05$ ), On CTSIB frim eyes closed and foam eyes closed show significant difference in both groups $(p$ $<0.05$ ). Between group analysis independent sample $T$ Test was applied and show that no statistically significant relation in both groups $(p$ $>0.05$ ).

Table-I was indicate descriptive statistics of BBS, TUG and CTSIB of Group A at baseline and after $8^{\text {th }}$ weeks.

Table-II indicates descriptive statistics of BBS, TUG and CTSIB of Group B at baseline and after $8^{\text {th }}$ weeks.

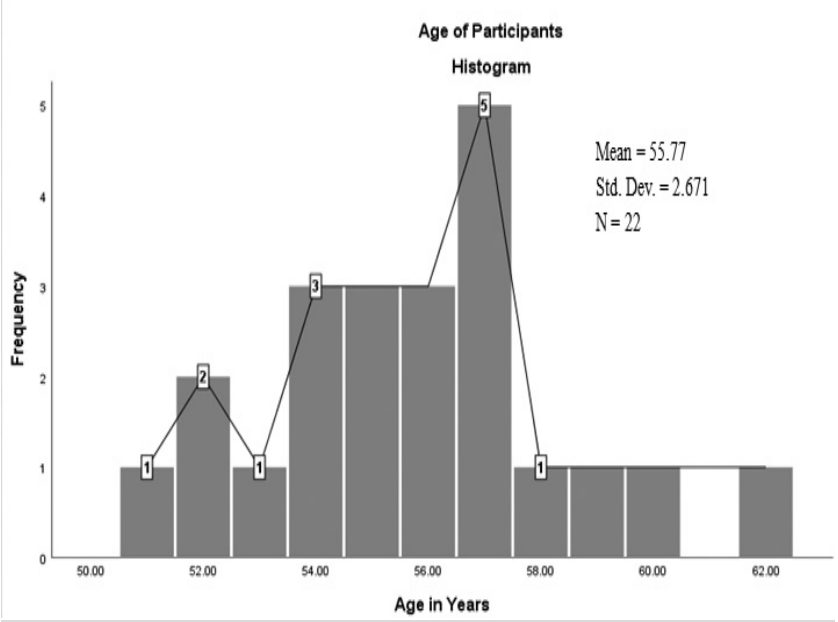

Figure-1. Shows frequency distribution of age of the participants

\section{Gender Distribution in Group A}

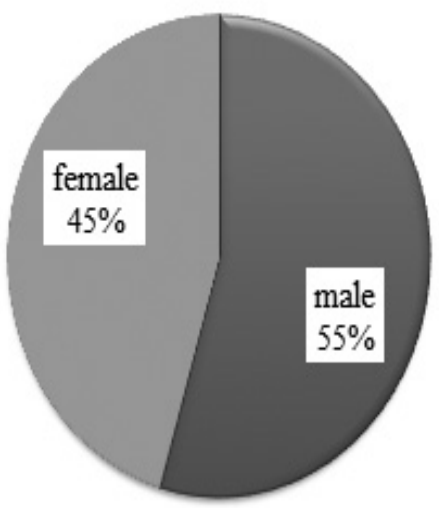

Figure-2 (A)

Gender Distribution in Group B

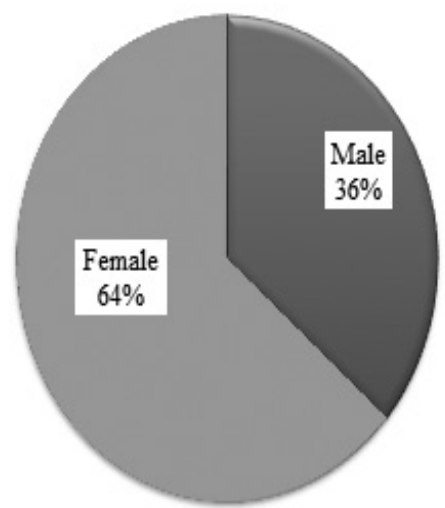

Figure-2. (B) 


\begin{tabular}{|l|c|c|c|}
\hline & Pre Observations & Post Observations & \\
\hline & Mean \pm Std. Deviation & Mean \pm Std. Deviation & .036 \\
\hline Berg Balance Score & $36.78 \pm 4.93$ & $44.44 \pm 3.609$ & .005 \\
\hline Time Up \&Go & $16.33 \pm 1.414$ & $11.00 \pm 2.000$ & .499 \\
\hline Firm: Eyes Open & $27.11 \pm 4.484$ & $29.67 \pm 1.000$ & .000 \\
\hline Firm: Eyes Closed & $16.67 \pm 4.500$ & $22.67 \pm 4.607$ & .043 \\
\hline Firm: Dome & $15.78 \pm 2.779$ & $19.00 \pm 4.358$ & .116 \\
\hline Foam: Eyes Open & $26.89 \pm 4.833$ & $29.22 \pm 1.715$ & .002 \\
\hline Foam: Eyes Closed & $14.33 \pm 3.937$ & $18.11 \pm 4.598$ & .153 \\
\hline Foam: Dome & $15.89 \pm 3.217$ & $16.11 \pm 6.607$ & \\
\hline & Table-I. Pre \& Post observations of Group A. & \\
\hline & Pre Observations & Post Observations & P-Value \\
\hline Berg Balance Score & Mean \pm Std. Deviation & Mean \pm Std. Deviation & .000 \\
\hline Time Up \&Go & $39.20 \pm 5.157$ & $45.70 \pm 3.093$ & .001 \\
\hline Firm: Eyes Open & $17.70 \pm 2.869$ & $11.40 \pm 2.756$ & .185 \\
\hline Firm: Eyes Closed & $26.30 \pm 4.854$ & $29.50 \pm 1.581$ & .000 \\
\hline Firm: Dome & $19.50 \pm 7.501$ & $23.70 \pm 5.056$ & .067 \\
\hline Foam: Eyes Open & $15.90 \pm 2.131$ & $19.40 \pm 5.601$ & .099 \\
\hline Foam: Eyes Closed & $25.10 \pm 6.436$ & $29.70 \pm 0.948$ & .002 \\
\hline Foam: Dome & $19.10 \pm 7.823$ & $21.40 \pm 7.381$ & .056 \\
\hline
\end{tabular}

Table-I. Pre \& Post Observations of Group B.

\section{DISCUSSION}

This study demonstrated that within groups comparison (Group A) mean and SD of berg balance scale $(44.4 \pm 3.6)$ shows statistically significant progress in BRACE group $(p=0.036)$ and (Group B) mean and of berg balance scale $(45.7 \pm 3.0)$ show statistically significant progress in proprioceptive training group $(p=0.000)$. RoigCasasus et al. in 2018 studied that a 4 week training platform whose approaches comprised dynamo-metric stand resulted in significance progress of experimental groups measured with Berg Balance Scale (51.8 \pm 2.7$),(p=0.03){ }^{21}$

Gulsen et al., in (2019) studied on older people aged 65 and over were given instrumental balance training with virtual reality practice. Result of the study was show an improvement in the balance in older people same as current study. Another study show that balance is impaired in knee osteoarthritis and the risk of falling is expected to increase. Falling occurs mostly during daily life activities situations that requiring dynamic postural control. ${ }^{8}$ In current study both training programs resulted in significant improvements in balance measured with berg balance scale. Doma et al. in 2018 determine the benefits of balance training on balance performance. Meta analyses showed that balance-specific training improved walking capacity. However the current meta-analyses verified that persons who undertook approximately 6 weeks of balance training as an assistant showed significantly greater improvement in balance specific tasks. ${ }^{22}$

In current study results also showed that 8 weeks both training programs give significant improvements in mobility measured with time up and go test. Mean and SD of BRACE group $(11.0 \pm 2.0)$ with significant value $(p=0.005)$ and mean and SD of Proprioceptive group (11.4 \pm 2.7$)$ with significant value $(p=0.001)$.

This results supported by literatue of Bishoff HA et al. who suggested that TUG score $<12$ was required to decrease risk of fall and improve mobility. They also suggested that the TUG test is useful in detecting mobility impairments in elderly 
persons. ${ }^{23} \mathrm{Ng}$ et al. in 2017 stated that TUG test is used to measure for perceiving change in mobility in older adults with high reliability. In exercise training goal the ankle was found to produce greater progress in postural stability. The intervention group also demonstrated a trend of an improvement in mobility and balance in single leg stance test. ${ }^{24}$

In this study CTSIB also shows significant improvement in two conditions frim eyes closed and foam eyes closed in both training groups. Other two conditions frim eyes open and foam eyes open proprioceptive training group is superior to BRACE group. Another condition of CTSIB frimdome BRACE group is superior to proprioceptive training group. Last condition of CTSIB foam dome proprioceptive training group is superior to BRACE group.

Hill et al., in 2013 verified high risk of falls in balance and mobility impairment in older women with OA showed most balance and mobility tasks in both groups of arthritis participants verified similar increased levels of across several CTSIB domains. ${ }^{25}$ Ricci et al. in 2009 defined elderly individuals with different histories of falls presented differences regarding the influence of sensory interaction on balance.Under some conditions in which proprioception was in accurate that was performed on foam. ${ }^{26}$

But in current study only foam in dome was not statistically significant. Overall sum of mean difference in BRACE group was more effective than proprioceptive group.

\section{CONCLUSION}

It is concluded that both groups revealed comparable improvements in balance and mobility but BRACE protocol showed more improvement as compared with proprioceptive.

Copyright@ 05 Jan, 2022.

\section{REFERENCES}

1. Topp R, Pifer M. A preliminary study into the effect of 2 resistance training modes on proprioception of subjects with knee osteoarthritis. Journal of Performance Health Research. 2017; 1(1):26.
2. Braghin RdMB, Libardi EC, Junqueira C, NogueiraBarbosa $\mathrm{MH}$, de Abreu DCC. Exercise on balance and function for knee osteoarthritis: A randomized controlled trial. Journal of bodywork and movement therapies. 2018; 22(1):76-82.

3. Beckwée D, Vaes P, Cnudde M, Swinnen E, Bautmans I. Osteoarthritis of the knee: Why does exercise work? A qualitative study of the literature. Ageing research reviews. 2013; 12(1):226-36.

4. Kim H-S, Yun DH, Yoo SD, Kim DH, Jeong YS, Yun J-S, et al. Balance control and knee osteoarthritis severity. Annals of rehabilitation medicine. 2011; 35(5):701.

5. Jyoti SJ, Sharma P. Role of proprioceptive exercises in knee osteoarthritis. Website: www ijpot com. 2017; 11(3):33.

6. Chae SH, Kim YL, Lee SM. Effects of phase proprioceptive training on balance in patients with chronic stroke. Journal of physical therapy science. 2017; 29(5):839-44.

7. UzunkulaoĞlu A, Kerim D, Saime AY, Ergin S. Effects of single-task versus dual-task training on balance performance in elderly patients with knee osteoarthritis. Archives of Rheumatology. 2020 Mar; 35(1):35.

8. Gulsen $M$. The effects of balance training by tetrax interactive balance system on fall risk in older women with knee osteoarthritis: A randomized controlled trial. 2019.

9. Ferraz RB, Gualano B, Rodrigues R, Kurimori CO, Fuller $R$, Lima FR, et al. Benefits of resistance training with blood flow restriction in knee osteoarthritis. Med Sci Sports Exerc. 2018; 50(5):897-905.

10. Jung K, Kim $\mathrm{Y}$, Chung $\mathrm{Y}$, Hwang S. Weight-shift training improves trunk control, proprioception, and balance in patients with chronic hemiparetic stroke. The Tohoku journal of experimental medicine. 2014; 232(3):195-9.

11. Messier SP, Beavers DP, Herman C, Hunter DJ, DeVita $P$. Are unilateral and bilateral knee osteoarthritis patients unique subsets of knee osteoarthritis? A biomechanical perspective. Osteoarthritis and cartilage. 2016; 24(5):807-13.

12. Singh AK, MAni KAIAivAni AK, AggArwAI PK, Gupta SK. Prevalence of osteoarthritis of knee among elderly persons in urban slums using American College of Rheumatology (ACR) criteria. Journal of clinical and diagnostic research: JCDR. 2014; 8(9):JC09. 
13. Huisingh C, Wadley VG, McGwin Jr G, Owsley C. Relationship between areas of cognitive functioning on the mini-mental state examination and crash risk. Geriatrics. 2018; 3(1):10.

14. Steffen TM, Hacker TA, Mollinger L. Age-and genderrelated test performance in community-dwelling elderly people: Six-Minute walk test, berg balance scale, timed up \& go test, and gait speeds. Physical therapy. 2002; 82(2):128-37.

15. Silva P, Botelho PFFB, de Oliveira Guirro EC, Vaz MMO, de Abreu DCC. Long-term benefits of somatosensory training to improve balance of elderly with diabetes mellitus. Journal of bodywork and movement therapies. 2015; 19(3):453-7.

16. Bogle Thorbahn LD, Newton RA. Use of the berg balance test to predict falls in elderly persons. Physical therapy. 1996; 76(6):576-83.

17. $\mathrm{Ng}$ SS, Hui-Chan $\mathrm{CW}$. The timed up \& go test: Its reliability and association with lower-limb impairments and locomotor capacities in people with chronic stroke. Archives of physical medicine and rehabilitation. 2005; 86(8):1641-7.

18. Lotfi $Y$, Javanbakht $M$, Sayaf M, Bakhshi E. Modified clinical test of sensory interaction on balance test use for assessing effectiveness of Epley maneuver in benign paroxysmal positional vertigo patients rehabilitation. Auditory and Vestibular Research. 2018; $27(1): 12-8$.

19. Franklin CB, Thomas VA, Dongho GBD, Faustin AT, Sharma KN. Conventional Physiotherapy And Additional Krishna's Kinetikinetic Manual Therapy (KKMT) for knee osteoarthritis rehabilitation: A comparative study. International Journal of Advanced Research and Publications. 2018; 2(1):54-8.
20. Santos SM, da Silva RA, Terra MB, Almeida IA, De Melo $\mathrm{LB}$, Ferraz $\mathrm{HB}$. Balance versus resistance training on postural control in patients with Parkinson's disease: A randomized controlled trial. European journal of physical and rehabilitation medicine. 2016; 53(2):17383.

21. Jogi P, Overend TJ, Spaulding SJ, Zecevic A, Kramer JF. Effectiveness of balance exercises in the acute post-operative phase following total hip and knee arthroplasty: A randomized clinical trial. SAGE open medicine. 2015 Feb 9;3:2050312115570769.

22. Doma K, Grant A, Morris J. The effects of balance training on balance performance and functional outcome measures following total knee arthroplasty: A systematic review and meta-analysis. Sports Medicine. 2018; 48(10):2367-85.

23. Bischoff HA, Stähelin HB, Monsch AU, Iversen MD, Weyh $A$, Von Dechend $M$, et al. Identifying a cut $\square$ off point for normal mobility: A comparison of the timed 'up and go'test in community $\square$ dwelling and institutionalised elderly women. Age and ageing. 2003; 32(3):315-20.

24. Ng TKW, Kwan RLC, Lo SK, Cheing GLY. A tailormade exercise program for improving balance and mobility in older adults with type 2 diabetes. Journal of gerontological nursing. 2017; 44(2):41-8.

25. Hill KD, Williams SB, Chen J, Moran H, Hunt S, Brand C. Balance and falls risk in women with lower limb osteoarthritis or rheumatoid arthritis. Journal of Clinical Gerontology and Geriatrics. 2013; 4(1):22-8.

26. Ricci NA, de Faria Figueiredo Gonçalves D, Coimbra AMV, Coimbra IB. Sensory interaction on static balance: A comparison concerning the history of falls of community $\square$ dwelling elderly. Geriatrics \& gerontology international. 2009; 9(2):165-71.

\begin{tabular}{|c|c|c|c|}
\hline \multicolumn{3}{|c}{ AUTHORSHIP AND CONTRIBUTION DECLARATION } \\
\hline No. & Author(s) Full Name & \multicolumn{1}{|c|}{ Contribution to the paper } & Author(s) Signature \\
\hline 1 & Ali Raza & $\begin{array}{l}\text { Introduction, Literature review, } \\
\text { Data collection \& analysis. } \\
\text { Supervision \& Data analysis. }\end{array}$ \\
\hline 2 & Khadija Liaquat & Delaze \\
\hline 3 & Hafiz Salman Saeed & Discussion. & \\
\hline
\end{tabular}

